\title{
DNA microarray analysis of global gene regulation by A-factor in Streptomyces griseus
}

\author{
Hirofumi Hara,† Yasuo Ohnishi and Sueharu Horinouchi \\ Department of Biotechnology, Graduate School of Agriculture and Life Sciences, University of \\ Tokyo, Bunkyo-ku, Tokyo 113-8657, Japan
}

Correspondence

Sueharu Horinouchi

asuhori@mail.ecc.u-tokyo.ac.jp

Received 26 January 2009

Revised 25 March 2009

Accepted 27 March 2009

\begin{abstract}
A-factor (2-isocapryloyl-3R-hydroxymethyl- $\gamma$-butyrolactone) is a microbial hormone that triggers morphological differentiation and secondary metabolism in Streptomyces griseus. The effects of A-factor on global gene expression were determined by DNA microarray analysis of transcriptomes obtained with the A-factor-deficient mutant $\triangle$ afs $A$. A-factor was added at a concentration of $25 \mathrm{ng} \mathrm{ml}^{-1}$ to mutant $\triangle a f s A$ at the middle of the exponential growth phase, and RNA samples were prepared from the cells grown after A-factor addition for a further 5, 15 and $30 \mathrm{~min}$, and 1, 2, 4, 8 and $12 \mathrm{~h}$. The effects of A-factor on transcription of all proteincoding genes of $S$. griseus were evaluated by comparison of the transcriptomes with those obtained from cells grown in the absence of A-factor. Analysis of variance among the transcriptomes revealed that 477 genes, which were dispersed throughout the chromosome, were differentially expressed during the $12 \mathrm{~h}$ after addition of A-factor, when evaluated by specific criteria. Quality threshold clustering analysis with regard to putative polycistronic transcriptional units and levels of upregulation predicted that 152 genes belonging to 74 transcriptional units were probable A-factor-inducible genes. Competitive electrophoretic mobility shift assays using DNA fragments including putative promoter regions of these 74 transcriptional units suggested that $\mathrm{AdpA}$ bound 37 regions to activate 72 genes in total. Many of these A-factor-inducible genes encoded proteins of unknown function, suggesting that the A-factor regulatory cascade of $S$. griseus affects gene expression at a specific time point more profoundly than expected.
\end{abstract}

\section{INTRODUCTION}

The Gram-positive, soil-inhabiting, filamentous bacterial genus Streptomyces is characterized by its ability to produce a wide variety of secondary metabolites represented by antibiotics and by its complex multicellular development. In Streptomyces griseus, both secondary metabolism and morphological development are triggered by a lowmolecular-mass chemical signalling molecule, A-factor (2-isocapryloyl-3R-hydroxymethyl- $\gamma$-butyrolactone)

\section{tPresent address: Department of Biomedical Engineering, Okayama} University of Science, Ridaicho, Kita-ku, Okayama 700-0005, Japan.

Abbreviations: ChIP-chip, chromatin immunoprecipitation chip; EMSA, electrophoretic mobility shift assay; GBL, $\gamma$-butyrolactone; PAG, probable A-factor-inducible gene; QT, quality threshold.

Details of the microarray design, transcriptome experimental design, and transcriptome data have been deposited in the NCBI Gene Expression Omnibus (GEO; http://www.ncbi.nlm.nih.gov/geo/) under accession number GSE13804.

Twelve supplementary tables, listing PCR primers used in this study, genes differentially expressed at various times after A-factor addition, and genes classified into clusters 1 and 2 , are available with the online version of this paper.
(Horinouchi, 2007; Horinouchi \& Beppu, 2007). A-factor is produced by the action of AfsA, which catalyses $\beta$ ketoacyl transfer from 8-methyl-3-oxononanoyl-acyl carrier protein to the hydroxyl group of dihydroxyacetone phosphate (Kato et al., 2007), and is gradually accumulated in a growth-dependent manner, reaching a maximum concentration of $25 \mathrm{ng} \mathrm{ml}^{-1}(100 \mathrm{nM})$ at or near the middle of the exponential growth phase (Ando et al., 1997). When the concentration of A-factor reaches a critical level, it binds the A-factor-specific receptor (ArpA) that was bound to the promoter of adpA and dissociates ArpA from the promoter, resulting in induction of adpA transcription (Onaka \& Horinouchi, 1997; Ohnishi et al., 1999). adpA is the sole target of ArpA to account for the biological effects of ArpA (Kato et al., 2004). The AraC/ XylS family transcriptional regulator AdpA then activates transcription of many genes that are required for secondary metabolism and morphological differentiation, forming an AdpA regulon (Ohnishi et al., 2005). Members of the AdpA regulon identified to date are $s t r R$, the pathway-specific transcriptional activator for streptomycin biosynthesis (Ohnishi et al., 1999; Tomono et al., 2005a); an ORF (SGR6071) encoding a probable pathway-specific transcriptional activator for biosynthesis of a polyketide 
compound (Yamazaki et al., 2004); adsA, encoding an extracytoplasmic function sigma factor of RNA polymerase essential for aerial mycelium formation (Yamazaki et al., 2000); $a m f R$, encoding a transcriptional activator for the amfTSBA operon responsible for production of a surfactant lantibiotic-like peptide SapB (AmfS) (Ueda et al., 2002, 2005), which is essential for aerial mycelium formation (Yamazaki et al., 2003b); ssgA, encoding a small acidic protein essential for spore septum formation (Yamazaki et al., 2003a); sgmA, encoding a secreted metalloendopeptidase probably involved in apoptosis of substrate mycelium during aerial mycelium development (Kato et al., 2002); five different genes (sprA, sprB, sprD, sprT and $s p r U$ ) encoding secreted serine proteases (Kato et al., 2005a; Tomono et al., 2005b); sgiA, encoding a Streptomyces subtilisin inhibitor (SSI) family inhibitor (Hirano et al., 2006); a gene of unknown function (SGR4618, formerly named orfA-AdBS3) (Yamazaki et al., 2004); and an operon of unknown function orf1-2-3-4 of AdBS2 (SGR6559-SGR6556) (Yamazaki et al., 2004). Thus, we have identified 14 AdpA target promoters to date.

Recently, we determined the complete genome sequence of S. griseus IFO13350 (Ohnishi et al., 2008). The linear chromosome consists of $8545929 \mathrm{bp}$, containing longterminal inverted repeats (TIRs) of $132910 \mathrm{bp}$ each. We predicted 7138 ORFs on the chromosome; 126 ORFs (SGR1t-SGR126t) in the left TIR sequence have identical counterparts (SGR7138t-SGR7013t) in the right TIR sequence. The genome sequence has enabled us to employ new approaches to analyse the A-factor regulatory cascade more comprehensively and extensively. One of the new approaches was transcriptome analysis using a DNA microarray. We prepared an oligonucleotide microarray, by which the transcripts of all 7138 ORFs could be theoretically monitored, and compared transcriptomes of the wild-type strain and an adpA-deleted $(\triangle a d p A)$ mutant in the early stationary phase in liquid culture (Ohnishi et al., 2008). The transcriptome analysis with certain criteria to discriminate the transcriptional level of a gene between the wild-type and mutant $\Delta a d p A$ strains showed that 639 genes were transcriptionally downregulated and 373 genes were upregulated in the $\triangle a d p A$ mutant (see Fig. 2, line b), predicting global gene expression by A-factor. However, it was probable that many side effects derived from physiological differences between the wild-type and $\Delta a d p A$ strains were also picked up in this experiment. In the present study, therefore, we attempted to identify A-factor-responsive genes by a more direct transcriptome analysis. We determined the effect of exogenously supplemented A-factor on the transcriptome of an A-factor-deficient mutant, $\triangle a f_{s} A$, in which $a f_{s} A$, encoding the key enzyme for A-factor biosynthesis, was deleted (Kato et al., 2007). The effects of Afactor on transcription of all ORFs of S. griseus were evaluated by DNA microarray analysis by a comparison of the transcriptomes with those obtained from cells grown in the absence of A-factor. This procedure enabled us to follow the time-course of the gene expression triggered by A-factor.
The purposes of this study were (i) to confirm the A-factor regulatory cascade proposed in our previous studies, (ii) to show the direct effect of A-factor on genome-wide gene expression, (iii) to find novel A-factor-inducible genes, and (iv) to find possible AdpA target genes among them.

\section{METHODS}

Culture conditions. S. griseus IFO13350 (=NBRC102592) was obtained from the Institute of Fermentation (Osaka, Japan). S. griseus mutant $\triangle a f_{S A}$ (Kato et al., 2007) was pre-cultured at $30{ }^{\circ} \mathrm{C}$ for 2 days in $100 \mathrm{ml}$ YMPD medium containing $0.5 \%$ glycine [in a $500 \mathrm{ml}$ shaking (Sakaguchi) flask] with reciprocal shaking at 120 r.p.m. YMPD is a nutrient-rich medium ( $\mathrm{pH} 7.2)$ consisting of $0.2 \%$ yeast extract, $0.22 \%$ meat extract, $0.4 \%$ bactopeptone, $0.5 \%$ $\mathrm{NaCl}, 0.2 \% \mathrm{MgSO}_{4} \cdot 7 \mathrm{H}_{2} \mathrm{O}$, and $1 \%$ glucose. A portion $(20 \mathrm{ml}$ per flask) of the pre-culture was inoculated into two 51 Erlenmeyer flasks with baffles, containing 21 YMPD medium with $0.5 \%$ glycine. After cultivation with rotary shaking at 150 r.p.m. at $30{ }^{\circ} \mathrm{C}$ for $12 \mathrm{~h}, \mathrm{~A}$ factor in $2.5 \mu \mathrm{l}$ ethanol was added at a final concentration of $25 \mathrm{ng}$ $\mathrm{ml}^{-1}$ to one flask, and $2.5 \mu \mathrm{l}$ ethanol as a negative control was added to the other flask. A portion of the culture $(20 \mathrm{ml})$ was taken after addition of A-factor or ethanol at 5, 15 and $30 \mathrm{~min}$, and 1, 2, 4, 8 and $12 \mathrm{~h}$, and the cells were harvested by centrifugation. For RNA isolation, RNAlater (Ambion) was added to the cells, and the mixture was incubated at $4{ }^{\circ} \mathrm{C}$ for $12 \mathrm{~h}$ according to the manufacturer's instructions. The cells were collected by centrifugation and stored at $-80{ }^{\circ} \mathrm{C}$ until RNA extraction. Three sets of RNA samples prepared at the eight time points were applied to the transcriptome analysis. For examination of growth, mutant $\Delta a f_{s A}$ was cultured under similar conditions; A-factor was added to the culture at the same time point $12 \mathrm{~h}$ after inoculation. A portion $(10 \mathrm{ml})$ of the culture was taken at several time points and its dry cell weight was measured. These operations were repeated three times.

Microarray hybridization and data analysis. Total RNA was extracted from cells stored at $-80{ }^{\circ} \mathrm{C}$, and cDNA was synthesized and labelled with $\mathrm{Cy} 3$ and $\mathrm{Cy} 5$, as described previously (Ohnishi et al., 2008). Microarray preparation and the method for microarray hybridization were also described previously (Ohnishi et al., 2008). Among the triplicate cDNA samples obtained from the A-factorsupplemented culture, two were labelled with Cy5 and the other one was labelled with $\mathrm{Cy} 3$. In contrast, among the triplicate cDNA samples obtained from the culture without A-factor, two were labelled with Cy3 and the other one was labelled with Cy5. Duplicate competitive hybridization experiments using the Cy5-labelled cDNA from the A-factor-supplemented culture plus the Cy3-labelled cDNA from the culture without A-factor, and a single competitive hybridization experiment using the Cy3-labelled cDNA from the Afactor-supplemented culture plus the Cy5-labelled cDNA from the culture without A-factor were carried out. Equal amounts (50 pmol) of Cy3- and Cy5-labelled cDNA were used in all hybridizations. The expression ratios (with A-factor: without A-factor) were normalized with the linear model for microarray analysis (LIMMA) loess (subgrid) method using ArrayPipe 2.0 (Hokamp et al., 2004). Normalized expression ratios were calculated for each gene and tested for significance with the criteria of an expression ratio of either $>2.0$ or $<0.5$ and $P<0.05$. Two or more specific oligonucleotides for each of the 5643 large ORFs and one oligonucleotide for each of the 1495 ORFs of small size were designed. When two or more oligonucleotides were prepared for an ORF, the expression ratios estimated from the respective oligonucleotides were not exactly the same, because the hybridization efficiencies of the respective oligonucleotides were different. This difference caused the difference in signal intensity and gave rise to variation. In such cases, between 
the respective expression ratios (change values), a change value with the lowest $P$ value in a statistical analysis [a $t$ test or analysis of variance (ANOVA)] was regarded as being most reliable and selected. Note that the change values in Supplementary Tables S10-S12 were occasionally different from those in Supplementary Tables S2-S9, because the $P$ values in the $t$ test (Supplementary Tables S2-S9) and in the ANOVA (Supplementary Tables S10-S12) were completely different, and different change values resulting from different oligonucleotide probes could be selected between the two statistical analyses. The TIGR-MEV package (http://www.jcvi.org/cms/research/ software/) was used for hierarchical clustering and quality threshold (QT) clustering analyses based on the Pearson correlation. We confirmed that there was a very good correlation between expression ratios measured using the DNA microarray and quantitative RT-PCR analyses for 17 randomly selected regulator genes (Ohnishi et al., 2008).

Competitive electrophoretic mobility shift assay (EMSA). An $\sim 800$ bp DNA fragment, which probably included an A-factorinducible promoter and its upstream regulatory sequence, was prepared by PCR. A primer set for amplification of the $800 \mathrm{bp}$ DNA fragment was designed using Primer3 (http://primer3.sourceforge.net/); a $1 \mathrm{~kb}$ DNA sequence from -700 to +300 (taking the first nucleotide of the start codon as +1 ) was used as the query. The amplified $800 \mathrm{bp}$ DNA fragments invariably included a $600 \mathrm{bp}$ sequence from -500 to +100 . The concentration of each of the DNA fragments was estimated by ethidium bromide staining after agarose gel electrophoresis. Recombinant AdpA protein with a His-tag at its $\mathrm{C}$ terminus was produced in Escherichia coli and purified by an Ni-NTA column, as described previously (Yamazaki et al., 2000). Two DNA fragments containing the AdpA-binding sites of $\operatorname{sgmA}$ and sgiA were labelled with digoxigenin (DIG) using the DIG DNA Labelling kit (Roche) and were used as probes. The $\operatorname{sgmA}$ probe ( $\operatorname{sgmA}-\mathrm{P})$ was $300 \mathrm{bp}$ long (nucleotide positions from -399 to -100 , taking the transcriptional start point as +1 ) and contained the AdpA-binding B site for sgmA (Kato et al., 2002). The sgiA probe (sgiA-P) was $209 \mathrm{bp}$ (nucleotide positions from -183 to +26 ) and contained the AdpAbinding site for sgiA (Hirano et al., 2006). Recombinant AdpA $(10 \mu \mathrm{g})$ was incubated with DIG-labelled $\operatorname{sgmA}-\mathrm{P}$ and $\operatorname{sgiA}-\mathrm{P}(5 \mathrm{ng})$ in the presence of a 10-fold excess of a competitor DNA fragment, $1 \mu \mathrm{g}$ poly $(\mathrm{dI}-\mathrm{dC})-(\mathrm{dI}-\mathrm{dC})$ and $0.4 \mathrm{mg}$ BSA for $30 \mathrm{~min}$ at room temperature in buffer $(40 \mu \mathrm{l})$ containing $50 \mathrm{mM} \mathrm{Na} 2 \mathrm{HPO}_{4}, 10 \mathrm{mM}$ Tris/ $\mathrm{HCl}(\mathrm{pH} 8.0)$ and $15 \%(\mathrm{v} / \mathrm{v})$ glycerol. The reaction mixture was then applied to $4 \%$ PAGE at $4{ }^{\circ} \mathrm{C}$ for $1.5-2 \mathrm{~h}$ at $25 \mathrm{~mA}$. The DNA was electroblotted onto a Hybond-N+ nylon membrane (Amersham) for $30 \mathrm{~min}$ at $200 \mathrm{~mA}$ in $0.5 \times$ Tris-borate-EDTA buffer $(44.5 \mathrm{mM}$ Tris/HCl, $44.5 \mathrm{mM}$ boric acid and $1 \mathrm{mM}$ EDTA, $\mathrm{pH}$ 8.0). For chemiluminescent detection, the DIG Luminescent Detection kit (Roche) was used. We rated the binding of AdpA to each DNA fragment in six ranks; -, no competition; +, competition only with sgiA-P; ++ , weak competition with $\operatorname{sgmA}-\mathrm{P} ;+++$, moderate competition with sgmA-P; ++++ , strong but incomplete competition with sgmA-P; and +++++ , complete competition with $\operatorname{sgm} A-\mathrm{P}$. All primers used in this study are listed in Supplementary Table S1.

\section{RESULTS}

\section{Exogenously added A-factor affects transcriptomes of the $\triangle$ afs $A$ mutant}

We performed transcriptome analysis using the S. griseus mutant $\Delta a f_{S} A$, which produces no A-factor due to the absence of the key A-factor biosynthetic enzyme AfsA
(Kato et al., 2007), but still responds to exogenously supplemented A-factor. Because the A-factor concentration reaches approximately $25 \mathrm{ng} \mathrm{ml}^{-1}(100 \mathrm{nM})$ at or near the middle of the exponential growth phase in the wild-type strain (Ando et al., 1997), the final concentration of Afactor supplemented to the culture of mutant $\triangle a f_{s} A$ was $25 \mathrm{ng} \mathrm{ml}^{-1}$. The time point of A-factor addition was near the middle of the exponential growth phase, as determined using a growth curve obtained by measuring dry cell weights (Fig. 1). The transcriptomes obtained with the RNA samples prepared from the mycelium grown for 5,15 and $30 \mathrm{~min}$, and 1, 2, 4, 8 and $12 \mathrm{~h}$ after the addition of Afactor were compared with those of a control culture without A-factor addition by competitive hybridization using DNA microarray analysis. The control culture contained the same amount of ethanol alone to compensate for the possible effect of ethanol.

In the DNA microarray analysis, we employed the following criteria to discriminate A-factor-responsive genes: those that showed an increase or decrease in expression of more than twofold and a $P$ value of $<0.05$ when analysed with a $t$ test $(n=3)$. When these criteria or thresholds were used, the following numbers of genes were transcriptionally up- and downregulated by A-factor addition: (i) at $5 \mathrm{~min}, 14$ and four genes, respectively; (ii) at $15 \mathrm{~min}, 12$ and four; (iii) at $30 \mathrm{~min}, 13$ and 14; (iv) at $1 \mathrm{~h}, 32$ and eight; (v) at $2 \mathrm{~h}, 72$ and 26; (vi) at $4 \mathrm{~h}, 118$ and 32; (vii) at 8 h, 229 and 33; and (viii) at 12 h, 275 and 57 (Fig. 2c and Supplementary Tables S2-S9). It was noteworthy that $a d p A$ was identified as a gene upregulated by A-factor at all eight time points: (i) 3.5 -fold $(P=0.004)$, (ii) 4.0-fold (0.004), (iii) 3.4-fold (0.010), (iv) 3.3-fold (0.005), (v) 3.1-fold (0.006), (vi) 2.8-fold (0.025), (vii) 4.2fold (0.006), and (viii) 3.0-fold (0.001). Thus, we could confirm that the transcription of adpA was quickly switched on by exogenously added A-factor and remained at an almost constant level for $12 \mathrm{~h}$ thereafter.

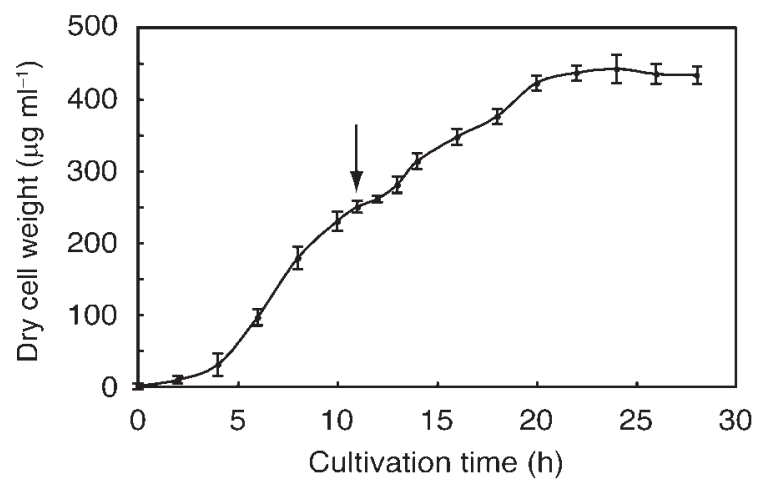

Fig. 1. Growth curve of $S$. griseus in YMPD medium. The arrow indicates the time point of A-factor addition, $12 \mathrm{~h}$ after inoculation. Each point shown represents the mean and SD of the values obtained from three independent experiments. 
(a)

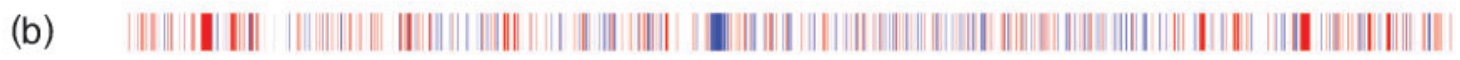

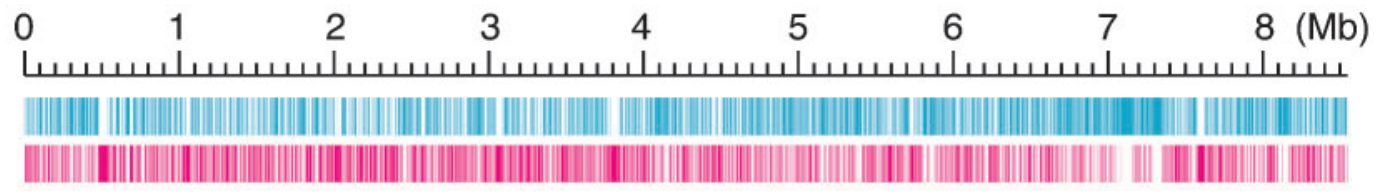

(c) i
$\mathrm{ii}$
$\mathrm{iii}$
$\mathrm{iv}$
$\mathrm{v}$
$\mathrm{vi}$
$\mathrm{vii}$
viii

(d)
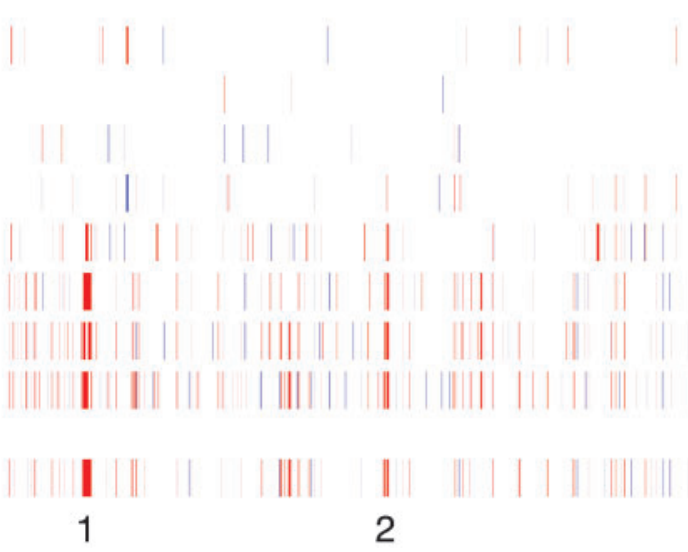

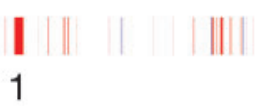

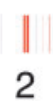
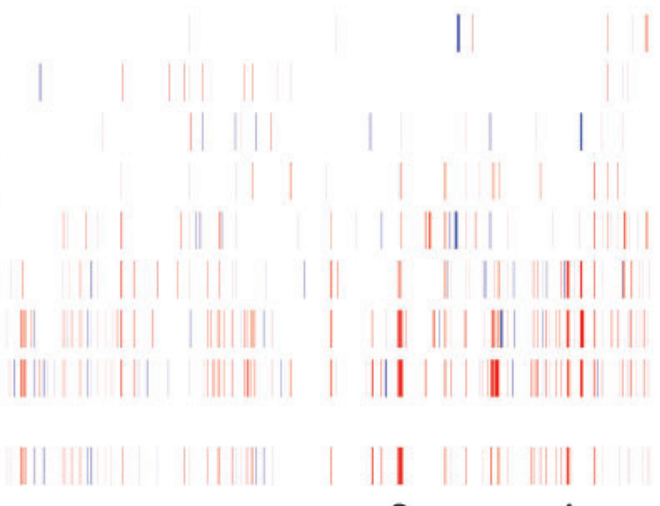

3

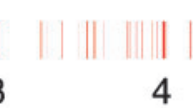

Fig. 2. Schematic representation of the transcriptomes obtained using RNAs prepared at eight different time points, i-viii. (a) Distribution of ORFs according to the direction of transcription (upper line, positive strand; lower line, negative strand). (b) Distribution of genes that were affected by adpA disruption, shown by our previous DNA microarray analysis $(P<0.05, n=4)$ (Ohnishi et al., 2008). Red, upregulated more than twofold in the wild-type strain compared with the $\Delta a d p A$ mutant; blue, downregulated more than twofold. (c) Distribution of genes that were affected by exogenously added A-factor, shown by the DNA microarray analysis $(P<0.05, n=3)$ at $5 \mathrm{~min}$ (i), $15 \mathrm{~min}$ (ii), $30 \mathrm{~min}$ (iii), $1 \mathrm{~h} \mathrm{(iv),} 2 \mathrm{~h} \mathrm{(v),} 4 \mathrm{~h} \mathrm{(vi),} 8 \mathrm{~h}$ (vii) and $12 \mathrm{~h}$ (viii) after A-factor addition. Red, upregulated more than twofold by A-factor addition; blue, downregulated more than twofold by A-factor addition. (d) Distribution of genes that were commonly detected in (b) and (c-viii). Four gene clusters are indicated as numerals below the line; 1, a putative NRPS gene cluster containing SGR452-SGR448; 2, a gene cluster of unknown function containing SGR2066-SGR2078; 3, the streptomycin biosynthesis gene cluster (SGR5914-SGR5940); 4, a putative NRPS gene cluster containing SGR6714-SGR6717.

\section{Genes upregulated within $\mathbf{3 0}$ min of A-factor addition}

In our previous study, we showed that adpA is the sole target of ArpA to account for the biological effects of ArpA (Kato et al., 2004). However, it was not clear whether or not ArpA directly represses other genes that could play important roles although the resulting phenotypes might not be observable. Putative ArpA-target genes, transcription of which must be repressed by ArpA in the $\Delta a f s A$ mutant, were assumed to be activated by exogenously added A-factor. Because the transcription of adpA was activated immediately after A-factor addition, the genes upregulated within $30 \mathrm{~min}$ after A-factor addition were considered to be possible candidates for putative ArpAtarget genes. Besides $a d p A, 13,11$ and 12 genes were detected as genes that were upregulated by A-factor at 5, 15 and $30 \mathrm{~min}$, respectively. However, in contrast to adpA, these genes were upregulated at only one time point during the $12 \mathrm{~h}$ period, except for six genes, SGR9t/SGR7130t (5 $\mathrm{min}$ and $2 \mathrm{~h}$ ), SGR2440 (30 $\mathrm{min}$ and $4 \mathrm{~h}$ ), SGR2794 (5 $\mathrm{min}$ and $12 \mathrm{~h}$ ), SGR3692 (5 $\mathrm{min}$ and $1 \mathrm{~h}$ ), SGR5078 (15 min and $1 \mathrm{~h}$ ), SGR6188 (30 min, and 1 and $2 \mathrm{~h}$ ) and
SGR6892 (5 min, and 1 and $2 \mathrm{~h}$ ), which were detected at two or three time points. In the three time points within $30 \mathrm{~min}$, no genes other than adpA were reproducibly detected. Although such transient activation seemed to be unlikely for genuine ArpA-target genes, we searched for an ArpA-binding consensus sequence, a $22 \mathrm{bp}$ palindromic sequence $\quad 5^{\prime}-(\mathrm{A} / \mathrm{C}) \mathrm{C}(\mathrm{A} / \mathrm{G})(\mathrm{T} / \mathrm{A}) \mathrm{ACCG}(\mathrm{A} / \mathrm{G}) \mathrm{CC}^{\star} \mathrm{GG}(\mathrm{T} /$ C)CGGT(A/T)(T/C)G(T/G)-3' (the asterisk indicates a dyad axis; essential nucleotides for ArpA binding are underlined), in the regions upstream from each of the candidate genes. As a result, only three genes, all of which encode hypothetical proteins, were found to have sequences similar to the consensus sequence in their upstream regions: $5^{\prime}$-ggAcACgGcCC ${ }^{\star}$ GGTCcGTggtG-3' $(-16$ to +6 , referring to the translational start point as +1 ; nucleotides that do not match with the consensus sequence are shown by small letters) for SGR23t/SGR7116t (5 min, 18.5-fold), 5'-CCGcACCGcCC ${ }^{\star}$ GGgttGTgatG-3' ( -237 to -216$)$ for SGR510 (5 min, 3.44-fold), and $5^{\prime}$ CgATACCtGCC $^{\star}$ GtTCGGTATtT-3' $^{\prime}(-217$ to -196$)$ for SGR5183 (30 min, 2.14-fold). It was noteworthy that we had cloned the DNA fragment containing the region upstream from SGR5183 as a DNA fragment bound by 
ArpA in vitro (Horinouchi et al., 2000). The presence of the putative ArpA-binding sequences suggested that these three genes are directly regulated by ArpA, although an unknown mechanism(s) is presumably required for their transient transcriptional activation. In contrast, the remaining early activated genes seemed not to be the direct targets of ArpA. It was unclear how exogenous A-factor triggered transient activation of these genes. Further analyses of these early activated genes, including three putative ArpA-target genes, could reveal novel aspects of the A-factor regulatory cascade. However, we focus below on the gene expression triggered by AdpA, because adpA proved to be the only gene that was continuously activated by A-factor immediately after its addition. Therefore, AdpA plays an absolute role as a transmitter and amplifier of the A-factor signal, and accordingly analysis of the AdpA regulon seemed to be the most important for understanding the A-factor regulatory cascade.

\section{There is a gradual increase in the A-factor effect on the transcriptome}

At $1 \mathrm{~h}$ after A-factor addition, we detected $s t r R$ as a gene that was upregulated by A-factor (2.6-fold), indicating that AdpA began to activate the transcription of its target genes at between 30 and $60 \mathrm{~min}$ in response to exogenously supplemented A-factor. In accordance with this, the number of upregulated genes increased gradually for 1$12 \mathrm{~h}$ after A-factor addition; 32, 72, 118, 229 and 275 upregulated genes were detected at $1,2,4,8$ and $12 \mathrm{~h}$, respectively, after A-factor addition. Although activation of some of these genes was apparently transient, many were successively upregulated, especially from 4 to $12 \mathrm{~h}$ after Afactor addition (Fig. 2c). These data show that the effect of exogenous A-factor on gene expression in the $\Delta a f_{s} A$ mutant was gradually increased through the function of AdpA as an amplifier of the A-factor signal. Therefore, we next analysed temporal changes in gene expression that are triggered by A-factor and presumably amplified by AdpA, as described below.

\section{QT clustering analyses of DNA microarray data}

To determine temporal changes in gene expression, we analysed the variance of the DNA microarray data that were obtained with the RNAs prepared at the eight time points. This variance analysis suggested that 477 genes were differentially expressed after addition of A-factor [expression ratio (with A-factor: without A-factor) of either $>2.0$ or $<0.5$ at at least one time point and ANOVA $P<0.05$ ] (Supplementary Table S10). We analysed these 477 genes by QT clustering analysis to classify them into groups according to their expression patterns. They were grouped into 15 clusters (Fig. 3a-o), although 24 genes were not assigned to any of the 15 clusters (Fig. 3p). In total, 345 genes $(72 \%)$ were assigned to two major groups, clusters 1 and 2. Cluster 1 contained 281 genes (59\%), the expression level of which almost continuously increased after exogenous addition of A-factor (Fig. 3a). On the other hand, cluster 2 contained 64 genes (13\%), the expression level of which almost continuously decreased after A-factor addition (Fig. 3b). We analysed genes of these two clusters by hierarchical clustering analysis (Fig. 4a, b). Fig. 4(a) and Fig. 4(b) visually represent the characteristic gene expression patterns of clusters 1 and 2, respectively.

\section{Activation of streptomycin biosynthesis genes by A-factor}

To verify that our ANOVA of the DNA microarray analysis could accurately detect temporal changes of gene expression triggered by A-factor, we examined the activation of streptomycin biosynthesis genes. Expression ratios (with Afactor: without A-factor) of all 27 genes in the streptomycin biosynthesis gene cluster (SGR5914-SGR5940) analysed by ANOVA were plotted (Fig. 5). This graph clearly shows that most of the streptomycin biosynthesis genes were, as expected, identified as genes that were upregulated by Afactor. Furthermore, activation of strR appeared to be followed by activation of the other streptomycin biosynthesis genes, in good agreement with the function of StrR not only as a transmitter of the A-factor signal to the gene cluster but also as a master regulator of the gene cluster. Of the 27 genes, 24 (all except SGR5930, SGR5928 and SGR5916) were classified into cluster 1 (Fig. 3a), suggesting that cluster 1 was composed of A-factor-inducible genes. SGR5930 and SGR5928 belong to the same transcriptional unit, strDEL (SGR5930-5928). Another gene of this transcriptional unit, SGR5929, was detected as an Afactor-inducible gene, but the level of upregulation was not very high (2.4-fold upregulation $12 \mathrm{~h}$ after A-factor addition). It is possible that the transcriptional activation of strDEL (SGR5930-5928) was rather low and we therefore failed to detect SGR5930 and SGR5928 as A-factorinducible genes. On the other hand, although transcriptional activation of strVW-stsGFED (SGR5915-5920) appeared to be very high, we failed to detect SGR5916 as an A-factor-inducible gene. Some fundamental error in detecting the SGR5916 transcription must have caused the failure. The fact that the $P$ value in a $t$ test for SGR5916 at each time point was very high, more than 0.6 in most cases, supported this assumption. Thus, we could confirm the signalling pathway from A-factor to the streptomycin biosynthesis genes, i.e. A-factor to ArpA to AdpA to StrR to streptomycin biosynthesis genes. We concluded that most temporal changes in gene expression could be monitored by our DNA microarray analysis, although a small population of the A-factor-inducible genes could not be detected by our experimental system.

\section{Selection of probable A-factor-inducible genes (PAGs)}

Because most A-factor-inducible genes appeared to be classified into cluster 1 (Supplementary Table S11), we further analysed each gene in cluster 1 to select PAGs. First, 

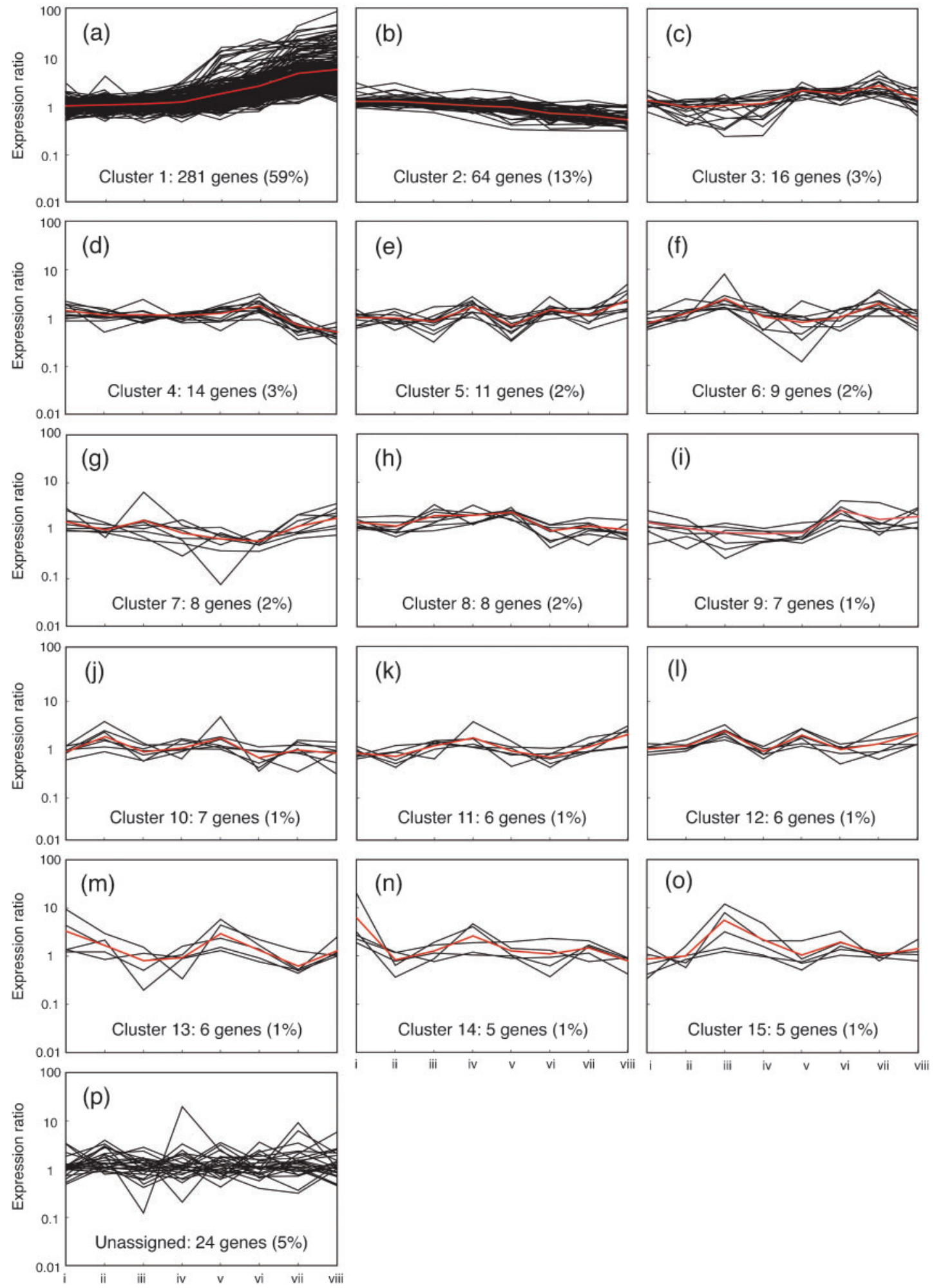

Fig. 3. QT clustering analysis of genes affected by exogenously added A-factor. The 477 genes with an increase or decrease in expression of more than twofold at at least one time point and an ANOVA $P<0.05$ were classified into 15 clusters (clusters $1-15, a-0)$, although 24 genes were not assigned to any of these 15 clusters (p). The average expression ratio (with Afactor: without $A$-factor) of all genes in each cluster (clusters 1-15) is shown in red. The time points are 5 min (i), 15 min (ii), $30 \mathrm{~min}$ (iii), $1 \mathrm{~h}$ (iv), $2 \mathrm{~h}$ (v), $4 \mathrm{~h}$ (vi), $8 \mathrm{~h}$ (vii) and $12 \mathrm{~h}$ (viii). 
(a)

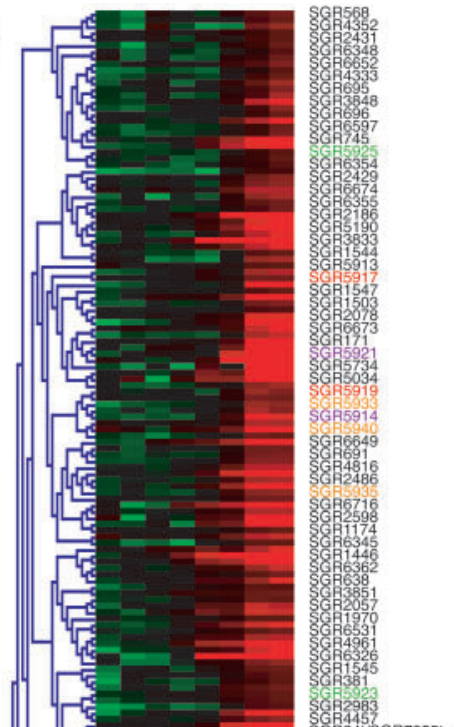

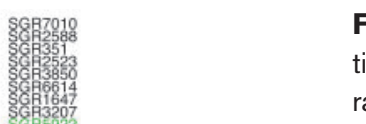

Fig. 4. Hierarchical clustering analysis of genes whose transcription was affected by exogenously added A-factor. The expression ratio (with $A$-factor: without $A$-factor) is shown in tones of red (positive values) or green (negative values). (a) Hierarchical clustering analysis of 281 genes that were classified into cluster 1 by the QT clustering analysis. The streptomycin biosynthesis genes are indicated in colours (see text for details). (b) Hierarchical clustering analysis of 64 genes that were classified into cluster 2 by the QT clustering analysis.

we gave careful consideration to the possible inclusion of the gene in question in a polycistronic structure. As described above, for example, we failed to detect SGR5916, a member of the strVW-stsGFED (SGR5915-5920) transcriptional unit, as an A-factor-inducible gene by DNA microarray analysis, although SGR5916 was apparently a true A-factor-inducible gene. Therefore, when only one or more genes in a putative polycistronic unit were not detected as A-factor-inducible genes, we regarded them as false negatives and added them to the PAGs. On the other hand, when only one gene in a putative polycistronic unit was detected as an A-factorinducible gene, it was a possible false positive and we did not consider it as a PAG. Second, we employed a stricter threshold for the selection of PAGs. Basically, when more than threefold upregulation on average at three time points or more than fourfold upregulation on average at two time points was observed, we regarded the gene as a PAG. However, when more than half the number of genes were detected as Afactor-inducible genes in a putative polycistronic unit, all members in the A-factor-inducible polycistronic unit were sometimes selected as PAGs, even if the upregulation of every gene in the unit was below the threshold. Using these criteria, careful examination identified 152 genes, belonging to 74 transcriptional units, as PAGs (Table 1).

In addition to the streptomycin biosynthesis genes, several genes probably involved in secondary metabolism were selected as PAGs: two putative non-ribosomal peptide synthetase (NRPS) gene clusters (SGR452-SGR448 and SGR6714-SGR6717), a lantibiotic biosynthesis gene cluster (SGR3851-SGR3845), putative melanin biosynthesis genes (SGR2446-2447) and a putative terpene cyclase gene (SGR2079). The previous DNA microarray analysis between the wild-type and $\triangle a d p A$ strains (Ohnishi et al., 2008) suggested that five secondary metabolism gene clusters [the streptomycin biosynthesis gene cluster, SGR443-SGR455 (NRPS), SGR574-SGR593 (NRPS), SGR6360-SGR6387 (type I polyketide synthase), and SGR6709-SGR6717 (NRPS)] were dependent on A-factor. Thus, in addition to the streptomycin biosynthesis gene cluster, two putative NRPS gene clusters were partly detected by both DNA microarray analyses (Fig. 2, line d). A further comparison of the two different DNA microarray analyses is included in the Discussion.

Only three genes that were probably involved in morphological development were selected as PAGs. SGR3340 


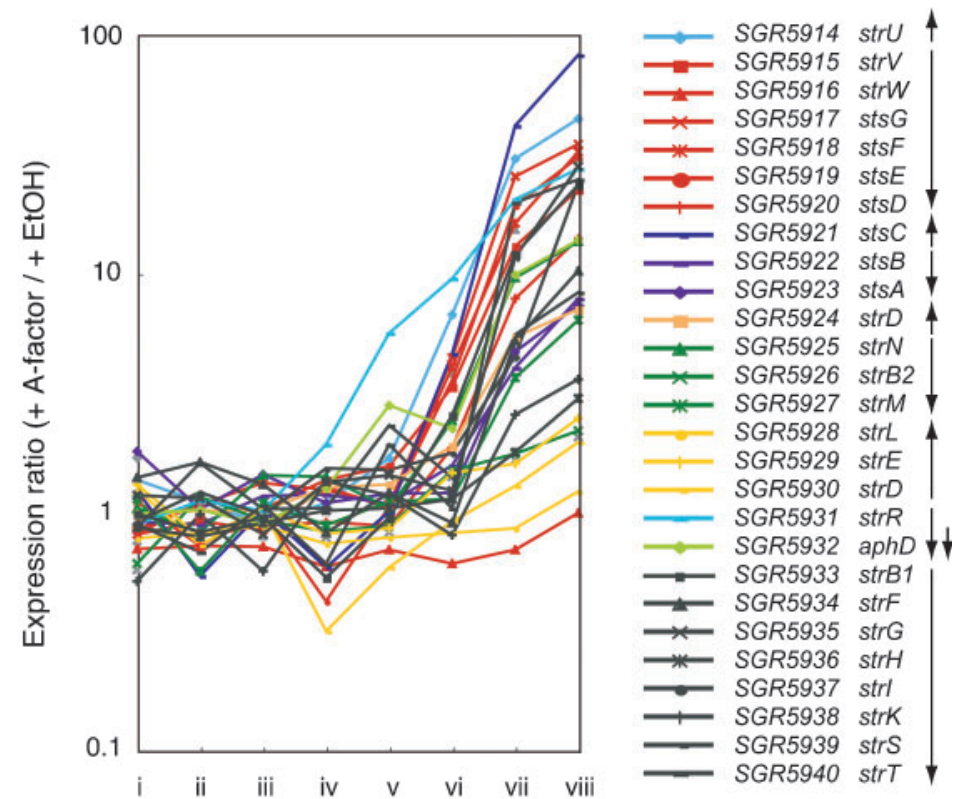

Fig. 5. Transcriptional upregulation of streptomycin biosynthesis genes by A-factor. Changes in the expression ratio (with $A$ factor: without A-factor) of all streptomycin biosynthesis genes are plotted. The ANOVA $P$ values of SGR5916, SGR5928 and SGR5930 are above the threshold (0.05). The ANOVA $P$ values of other genes are $<0.05$, indicating the reliability of the data. Organization of the transcriptional units in the gene cluster is schematically represented by arrows on the right of the gene names.

( $w b l A)$ has an important role in aerial mycelium formation in S. griseus (S. Hirano and others, unpublished data). SGR3902 (ORF1590) was cloned and characterized as a gene that could suppress a morphological defect of bald mutants of S. griseus (McCue et al., 1996). SGR299 encodes a Spo0M family protein. Although Spo0M in Bacillus subtilis exerts certain negative effects on sporulation (Han et al., 1998), the functions of SGR299 and its orthologues in Streptomyces remain to be characterized. We assume that the small number of PAGs involved in morphological development can be ascribed to differences in the mechanism or pattern of gene expression between liquid and solid medium. Although S. griseus can produce submerged spores in liquid medium (Kendrick \& Ensign, 1983), and A-factor is essential for submerged spore formation (Kawamoto et al., 1997; our unpublished observation), the mechanism of submerged spore formation is thought to be different from that of aerial spores. Therefore, many developmental genes that have been characterized using a solid culture seem to be undetectable in the liquid culture used in this study. In our previous DNA microarray analysis between the wild-type and $\triangle a d p A$ strains grown in liquid culture, many known AdpA target genes were not detected, but they were all detected in a similar analysis using the cells grown on solid medium (Ohnishi et al., 2008; our unpublished results).

Several PAGs probably have regulatory functions (Table 1). The in vivo functions of the A-factor-dependent DNAbinding proteins and conservons are worth characterizing. Conservons are paralogous sets of four (or five) genes encoding membrane-bound complexes that resemble eukaryotic G protein-coupled regulatory systems (Komatsu et al., 2006). Several putative enzyme genes and putative genes for transport and stress responses were also selected as PAGs (Table 1). In addition to these genes, many gene clusters and genes of unknown function were selected as PAGs (Table 1). A large gene cluster (SGR2066SGR2078) encoding several phage-related proteins was highly activated by A-factor. This gene cluster was also detected as a putative AdpA-dependent gene cluster by the previous DNA microarray analysis between the wild-type and $\Delta a d p A$ strains (Ohnishi et al., 2008). It is possible that this gene cluster originated from a prophage, but its actual function is not clear. Two convergent polycistronic units (SGR6353-SGR6359 and SGR6365-SGR6360), which were also highly activated by A-factor, may be involved in the production of a structurally unknown secondary metabolite. Because these gene clusters are specific to S. griseus, they may be responsible for some characteristics of $S$. griseus. On the other hand, some of the hypothetical proteins encoded by PAGs are highly conserved among Streptomyces species (Table 1). These proteins may play important roles in morphological and physiological differentiation of Streptomyces.

\section{Genes downregulated by A-factor}

The DNA microarray analysis revealed that transcription of some genes decreased almost continuously after A-factor addition. The genes downregulated by $\mathrm{A}$-factor were classified into cluster 2 . The number of genes in cluster 2 (64 genes) was much smaller than that in cluster 1 (281 genes). Furthermore, the expression ratio (without Afactor: with A-factor) of almost all genes classified in cluster 2 was less than 3.0. These results suggested that the negative effect of A-factor on gene expression was smaller than the positive effect. We assumed that transcription of genes in cluster 2 decreased in response to changes in some physiological conditions triggered by A-factor. However, we could not completely exclude the possibility that these 
Table 1. PAGs determined in this study

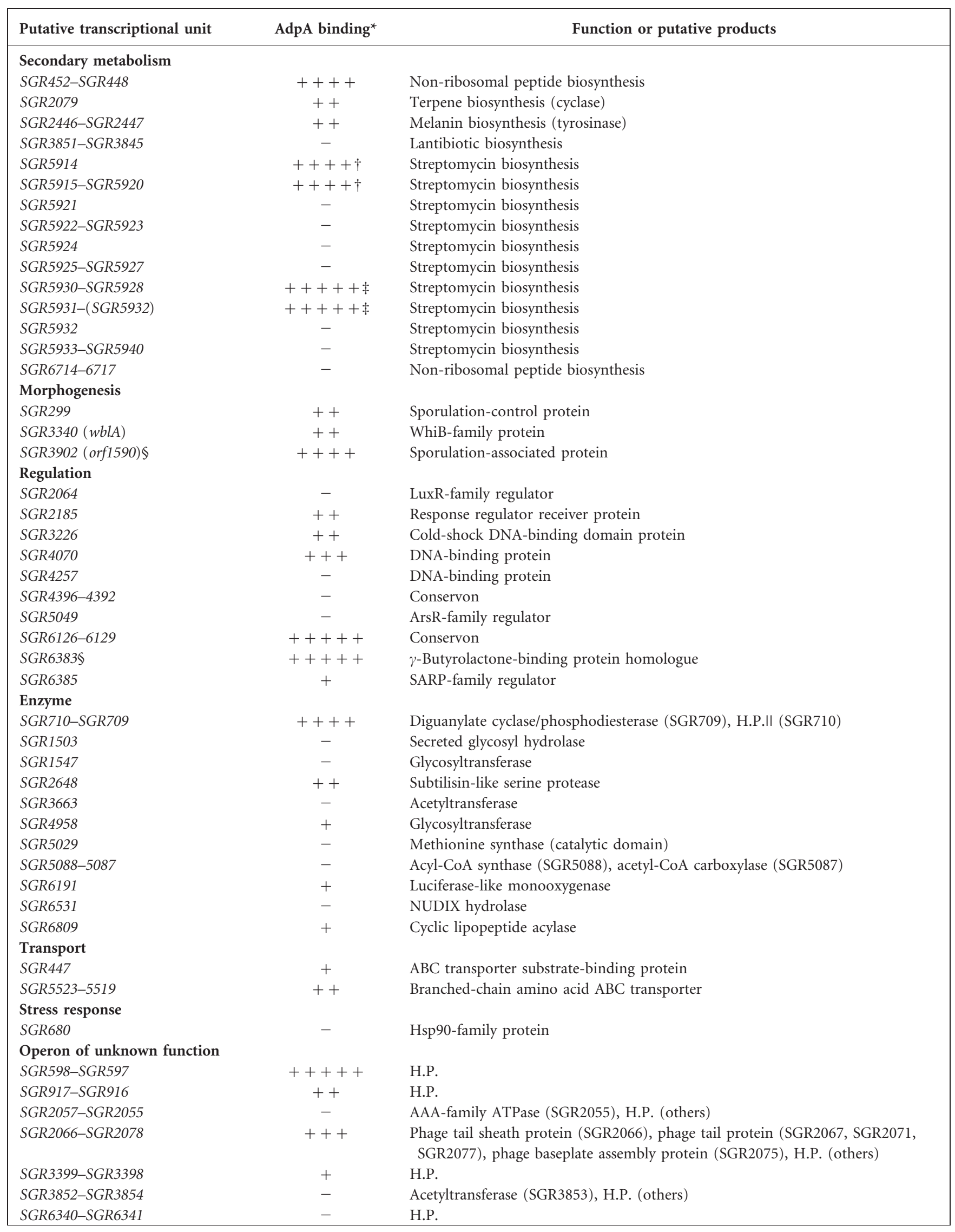


Table 1. cont.

\begin{tabular}{|c|c|c|}
\hline Putative transcriptional unit & AdpA binding* & Function or putative products \\
\hline SGR6353-SGR6359 & - & H.P. \\
\hline SGR6365-SGR6360 & + & $\begin{array}{l}\text { ATP-binding protein of ABC transporter (SGR6361), methyltransferase } \\
\text { (SGR6362), flavoprotein (SGR6363), H.P. (others) }\end{array}$ \\
\hline SGR6559-SGR6556 & + & AdBS2 ORF1-4 (H.P.) \\
\hline \multicolumn{3}{|l|}{ Unknown } \\
\hline SGR36t/SGR7103t & +++ & H.P. \\
\hline SGR165 & - & H.P. \\
\hline SGR296 & ++ & H.P. (highly conserved) \\
\hline SGR381 & - & H.P. \\
\hline SGR1002 & ++ & H.P. \\
\hline SGR1548 & - & H.P. \\
\hline SGR2065 & - & H.P. (conserved) \\
\hline SGR2148 & ++ & H.P. (highly conserved) \\
\hline SGR2456 & ++ & H.P. (conserved) \\
\hline SGR2532 & +++ & H.P. (conserved) \\
\hline SGR3144 & - & H.P. \\
\hline SGR3575 & ++ & H.P. \\
\hline SGR3840 & ++ & H.P. (highly conserved) \\
\hline SGR4457 & - & H.P. (highly conserved) \\
\hline SGR4961 & - & H.P. (highly conserved) \\
\hline SGR5190 & - & H.P. (highly conserved) \\
\hline SGR5529 & - & H.P. (highly conserved) \\
\hline SGR5941 & - & H.P. (conserved) \\
\hline SGR6153 & ++ & H.P. (highly conserved) \\
\hline SGR6856 & ++++ & H.P. (conserved) \\
\hline SGR6959 & - & H.P. (conserved) \\
\hline SGR7011 & ++ & H.P. \\
\hline
\end{tabular}

*The binding of AdpA to each DNA fragment is rated in six ranks as described in the text.

$\dagger$ The importance of binding of AdpA to the intergenic region between SGR5914 (strU) and SGR5915 (strV) was questionable, as described in the text.

¥The binding of AdpA to the intergenic region between SGR5930 (strD) and SGR5931 (strR) activates the latter but not the former.

§Two genes that do not fulfil the requirements for selection of PAGs but were exceptionally considered PAGs because of the function of interest. IH.P., hypothetical protein. When an H.P. has an end-to-end homology to a protein, it is shown as 'conserved'. When an H.P. is conserved among three Streptomyces species, S. griseus, Streptomyces coelicolor A3(2) and Streptomyces avermitilis ( $>70 \%$ identity or E value $<\mathrm{e}^{-100}$ in a BLAST search), it is shown as 'highly conserved'.

genes were repressed directly by AdpA, because AdpA acts as a transcriptional repressor on its own promoter (Kato et al., 2005b). Of 64 genes in cluster 2 (Supplementary Table S12), 27 encode proteins that are probably involved in the transport of metabolites. When morphological and physiological differentiation proceeds, biosynthesis of a variety of permeases and $\mathrm{ABC}$ transporters, which are probably required for vegetative growth, may decline.

\section{Putative members of the AdpA regulon}

As described above, we selected 152 genes belonging to 74 transcriptional units as PAGs. To estimate how many promoters are directly activated by AdpA, binding of AdpA to $\sim 800$ bp DNA fragments, which probably included a promoter and its upstream regulatory sequence for each Afactor-inducible gene, was determined by competitive
EMSA as described in Methods. We confirmed by preliminary tests that all of the $\sim 800$ bp DNA fragments that were designed similarly for 13 known AdpAdependent genes could be detected as AdpA-binding DNA fragments by the competitive EMSA (Fig. 6). Even when a DNA fragment had a low affinity for AdpA, we could detect sufficient competition of the DNA fragment against sgiA-P (Fig. 6b), but not against sgmA-P (Fig. 6a). sgmA-P has a much higher affinity for AdpA than sgiA-P. We rated the binding strength of AdpA to each DNA fragment in six ranks $(-,+,++,+++,++++$ and $+++++)$ on the basis of results of competitive EMSA using two DIG-labelled probes with different affinities for AdpA (Table 1). Our previous experiments suggested that the in vitro affinities of AdpA for its target DNAs do not reflect the level of in vivo transcriptional activation of the target genes. For example, AdpA binds to the region 
(a)

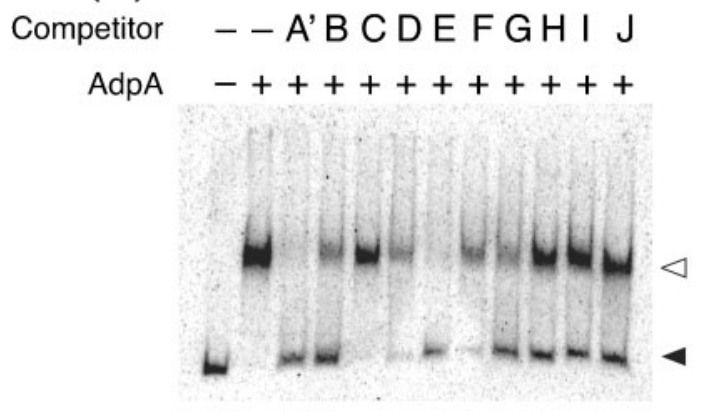

Probe: sgmA-P

(b)

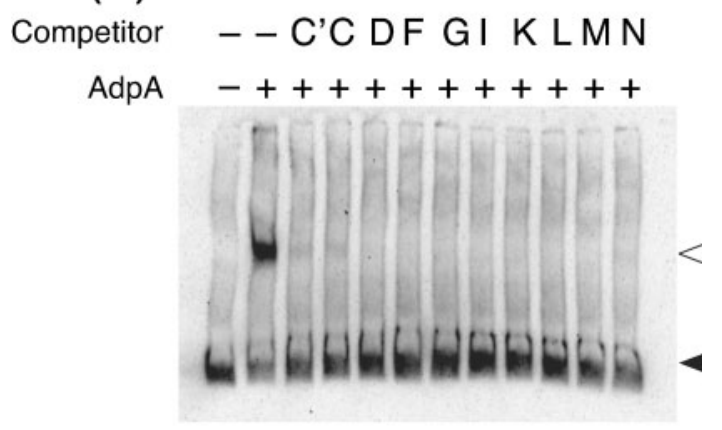

Probe: sgiA-P between SGR5914 (strU) and SGR5915 (strV), which should contain two divergent promoters of the two genes. Because an StrR-binding sequence was found in this region, and the promoters were inactive in an strR-deleted mutant (Tomono et al., 2005a), the importance of the AdpA binding to the region was questionable. Therefore, we excluded the region and estimated that AdpA should bind directly to 37 regions to activate 72 genes. Thus, approximately half of the promoters of PAGs appear to be activated directly by AdpA. Furthermore, the remaining promoters of PAGs appear to be activated secondarily by an AdpA-dependent transcription factor. Alternatively, these promoters may be activated in response to a change in certain physiological conditions triggered by A-factor.

\section{DISCUSSION}

Streptomyces employ $\gamma$-butyrolactones (GBLs) as chemical signalling molecules or microbial hormones for synchronizing gene expression in their complex mycelial networks. In general, many GBLs seem to act as a switch only for secondary metabolism, while some GBLs seem to affect the growth rate in addition to morphological differentiation and secondary metabolism (Takano, 2006). In other words, GBLs in Streptomyces function at different levels in the regulatory hierarchy of gene expression involved in vegetative growth, secondary metabolism and morphological differentiation. A-factor apparently controls an upper stage of the regulatory hierarchy involved in secondary metabolism and morphological differentiation, without affecting vegetative growth, as a strict switch, not as a tuner. Therefore, a gene expression profile triggered by A-factor represents an aspect of the transition of gene expression in the morphological and physiological differentiation of Streptomyces. In the present study, we showed the effects of exogenously supplemented A-factor on the transcriptome in the $\Delta a f s A$ mutant, which is deficient in Afactor production but still responds to A-factor.

Our previous DNA microarray analysis between the wildtype and $\triangle a d p A$ strains suggested that 639 and 373 genes, respectively, were transcriptionally down- and upregulated (expression ratio of $>2.0$ or $<0.5$ and $P$ of $<0.05$ ) in the $\triangle a d p A$ strain in the early stationary phase (Ohnishi et al., 2008) (see Fig. 2, line b). Thus, a total of 988 genes, apart from a duplication of 24 genes located on TIRs, appear to be affected by the absence of AdpA. When these data were compared with the results of the present study (275 upregulated and 57 downregulated genes at $12 \mathrm{~h}$ after Afactor addition; the cells were in the early stationary phase), 164 and 15 genes were shown to be commonly up- and downregulated, respectively, by the A-factor signal (Fig. 2, line d). Many side effects derived from physiological differences between the wild-type and $\Delta a d p A$ strains were presumably included in the result of the previous DNA microarray analysis. For example, the lag phase of the wildtype strain was approximately $3 \mathrm{~h}$ longer than that of mutant $\triangle a d p A$, probably due to the difference in 
physiological conditions of the inoculated cells; also, some wild-type cells produced submerged spores in the preculture but mutant $\triangle a d p A$ cells did not. Furthermore, the maximum cell mass of the $\triangle a d p A$ mutant in the culture was reproducibly smaller than that of the wild-type strain. These differences led to somewhat different growth of the two strains and made a comparison of their transcriptomes complicated. However, there were no such difficulties in the present DNA microarray analysis. We used only one strain and compared transcriptomes between the culture with A-factor addition and a negative control culture without A-factor addition at eight different time points to analyse temporal changes in gene expression triggered by A-factor. Therefore, the data obtained from the present DNA microarray analysis seem to reflect more direct effects of the deficiency in the A-factor regulatory cascade on the transcriptome than those obtained from the previous DNA microarray analysis. We finally selected 152 genes belonging to 74 transcriptional units as PAGs. Of the 152 genes, 136 were actually detected by the DNA microarray analysis and classified in cluster 1 in QT clustering analysis. Of the 136 genes, $103(76 \%)$ had been identified as AdpAdependent genes in the previous DNA microarray analysis between the wild-type and $\triangle a d p A$ strains, which indicates that the procedure for selection of PAGs in the present study is plausible.

Competitive EMSA, in which binding of AdpA to approximately $800 \mathrm{bp}$ DNA fragments containing a $5^{\prime}$ portion of each PAG with its upstream region was determined, showed that AdpA binds to 37 regions to activate 72 genes, including two known AdpA-dependent transcripts [strR-aphD (Vujaklija et al., 1991) and orf1-orf4 of AdBS2 (Yamazaki et al., 2004)]. The 200 bp DNA fragments were designed in such a way that they included a $600 \mathrm{bp}$ sequence from -500 to +100 (taking the first nucleotide of the start codon as +1 ). Although we did not determine the transcriptional start point of each PAG, the region presumably contained a promoter and its upstream regulatory sequence. This was supported by the observation that all of the $800 \mathrm{bp}$ DNA fragments designed similarly for 13 known AdpA-dependent genes were detected as AdpA-binding DNA fragments by competitive EMSA (Fig. 6). Thus, our method to screen for putative AdpA-dependent promoters seems to be appropriate, although it is possible that AdpA binding detected by competitive EMSA does not necessarily cause the apparent transcriptional activation of PAGs. Further analyses of the 34 candidates for novel AdpA-dependent promoters by determination of the exact AdpA-binding sites and the transcriptional start points are, for example, required to confirm that AdpA directly activates transcription from these promoters. To reveal a complete picture of the AdpA regulon, a combination of transcriptome analysis and analysis of in vivo AdpA-binding sequences is essential. Chromatin immunoprecipitation chip (ChIP-chip) analysis to identify in vivo AdpA-binding sequences is now in progress in our laboratory.
A-factor is gradually accumulated in a growth-dependent manner, reaching its maximum concentration of $\sim 25 \mathrm{ng}$ $\mathrm{ml}^{-1}(100 \mathrm{nM})$ at or near the middle of the exponential growth phase (Ando et al., 1997). In the present study, we abruptly added A-factor at a final concentration of $25 \mathrm{ng}$ $\mathrm{ml}^{-1}$ to mutant $\triangle a f_{s} A$ at the middle of the exponential growth phase. Therefore, the growth of mutant $\Delta a f_{S} A$ in the medium with exogenously added A-factor differed from that of the wild-type strain, where A-factor is gradually accumulated. However, we assume that the gene expression triggered by A-factor in mutant $\triangle a f_{S A} \mathrm{~A}$ is similar to that in the wild-type strain because A-factor seems to function when its concentration is above a critical level. adpA transcription was quickly triggered by $\mathrm{A}$-factor addition within $5 \mathrm{~min}$. In contrast to this rapid induction of $a d p A$, it took $1 \mathrm{~h}$ for activation of $s t r R$, an AdpA target gene. Because adpA contains a rare codon, TTA, translation of adpA mRNA may take a much longer time than that of the usual mRNAs with no TTA codons. The tRNA for the TTA codon is encoded by bldA, which is a developmentally significant gene (Chandra \& Chater, 2008). Thus, the presence of a TTA codon in adpA is one possible explanation of the apparent delay in transcriptional activation of the AdpA regulon after A-factor addition, although no transcriptional analysis of bldA in S. griseus has yet been performed. On the other hand, it is also possible that another transcription factor(s) is involved in the activation of some genes of the AdpA regulon, because upregulation of many putative AdpA-dependent promoters began to be detected at different time points between 1 and $4 \mathrm{~h}$ after $\mathrm{A}$-factor addition. A great difference in the timing of transcriptional activation has been detected among some known AdpA target promoters, such as strR (Tomono et al., 2005a), sprA (Tomono et al., 2005b) and sprT (Kato et al., 2005a), although these were analysed using RNA isolated from cells grown on solid medium. It is also possible that the difference in in vivo affinity of AdpA for each AdpA-dependent promoter accounts for these time lags. We previously showed that AdpA represses its own transcription as a transcriptional repressor (Kato et al., 2005b). However, the transcription of adpA was almost constant during the $12 \mathrm{~h}$ after A-factor addition, as determined by the DNA microarray analysis. Therefore, the function of AdpA as a repressor for its own transcription seems not to be exerted within $12 \mathrm{~h}$. However, it remains to be investigated whether the Afactor-dependent transcriptional repression detected by the present DNA microarray analysis can be ascribed to direct AdpA binding. ChIP-chip analysis of in vivo AdpA-binding sequences would assist such an investigation.

Most of the streptomycin biosynthesis genes were detected as genes grouped in cluster 1 by QT clustering analysis of the DNA microarray data. Because the streptomycin biosynthesis genes consist of 10 transcriptional units (see Fig. 5), the genes are appropriate for verification of the hierarchical tree of the genes in cluster 1 (Fig. 4a). Five (SGR5915 and SGR5917-SGR5920; shown in red in Fig. 4a) 
of six genes belonging to strVW-stsGFED were located close together in the tree. SGR5916 was not detected as a PAG by the DNA microarray analysis. However, three (SGR5934, SGR5938 and SGR5939; shown in blue in Fig. 4a) of eight genes belonging to strFGHIKST were located apart from the clade that included the other five genes (SGR5933, SGR5935-SGR5937 and SGR5940; shown in orange in Fig. 4a). This clade contains the five genes strVW-stsGFED described above and four other genes (SGR5914, SGR5921, SGR5924 and SGR5927; shown in purple in Fig. 4a) belonging to different transcriptional units. This result showed that more than half of the streptomycin biosynthesis genes classified into cluster 1 are located in the same clade in the hierarchical tree. Therefore, the hierarchical tree seems to reflect the actual gene expression pattern to some extent. However, other streptomycin biosynthesis genes (shown in green in Fig. 4a) were dispersed in the tree. This seems to be a limitation of the accuracy of our DNA microarray analysis.

As described above, it is clear that there are some limitations to our DNA microarray analysis. Using this analysis, however, we detected a direct influence of Afactor on genome-wide gene expression for what is believed to be the first time. We revealed that A-factor activates the transcription of many genes of unknown function that encode hypothetical proteins, suggesting that the A-factor regulatory cascade of $S$. griseus is more profound and complex than expected. To avoid overestimating the results of the DNA microarray analysis, we selected PAGs using several statistical analyses, taking into consideration a putative transcriptional unit containing the gene in question. Characterization of novel PAGs will provide much information about secondary metabolism and morphological differentiation in Streptomyces. Transcriptome analysis of cells grown on solid medium and ChIPchip analysis of in vivo AdpA-binding sequences are in progress in our laboratory, and should reveal several new aspects of global gene regulation by the A-factor regulatory cascade.

\section{ACKNOWLEDGEMENTS}

We thank N. Nakata for technical assistance in the competitive EMSA. This research was supported by a Grant-in-Aid for Scientific Research on Priority Area 'Applied Genomics' from the Ministry of Education, Culture, Sports, Science, and Technology of Japan, a research grant from the Noda Institute for Scientific Research, and a research grant from the Waksman Foundation of Japan.

\section{REFERENCES}

Ando, N., Ueda, K. \& Horinouchi, S. (1997). A Streptomyces griseus gene (sgaA) suppresses the growth disturbance caused by high osmolality and a high concentration of A-factor during early growth. Microbiology 143, 2715-2723.

Chandra, G. \& Chater, K. F. (2008). Evolutionary flux of potentially bldA-dependent Streptomyces genes containing the rare leucine codon TTA. Antonie Van Leeuwenhoek 94, 111-126.
Han, W. D., Kawamoto, S., Hosoya, Y., Fujita, M., Sadaie, Y., Suzuki, K., Ohashi, Y., Kawamura, F. \& Ochi, K. (1998). A novel sporulationcontrol gene (spoOM) of Bacillus subtilis with a $\sigma^{\mathrm{H}}$-regulated promoter. Gene 217, 31-40.

Hirano, S., Kato, J., Ohnishi, Y. \& Horinouchi, S. (2006). Control of the Streptomyces subtilisin inhibitor gene by AdpA in the A-factor regulatory cascade in Streptomyces griseus. J Bacteriol 188, 6207-6216.

Hokamp, K., Roche, F. M., Acab, M., Rousseau, M. E., Kuo, B., Goode, D., Aeschliman, D., Bryan, J., Babiuk, L. A. \& other authors (2004). ArrayPipe: a flexible processing pipeline for microarray data. Nucleic Acids Res 32, W457-W459.

Horinouchi, S. (2007). Mining and polishing of the treasure trove in the bacterial genus Streptomyces. Biosci Biotechnol Biochem 71, 283299.

Horinouchi, S. \& Beppu, T. (2007). Hormonal control by A-factor of morphological development and secondary metabolism in Streptomyces. Proc Jpn Acad Ser B Phys Biol Sci 83, 277-295.

Horinouchi, S., Onaka, H., Yamazaki, H., Kameyama, S. \& Ohnishi, Y. (2000). Isolation of DNA fragments bound by transcriptional factors, AdpA and ArpA, in the A-factor regulatory cascade. Actinomycetologica 14, 37-42.

Kato, J. Y., Suzuki, A., Yamazaki, H., Ohnishi, Y. \& Horinouchi, S. (2002). Control by A-factor of a metalloendopeptidase gene involved in aerial mycelium formation in Streptomyces griseus. J Bacteriol 184, 6016-6025.

Kato, J. Y., Miyahisa, I., Mashiko, M., Ohnishi, Y. \& Horinouchi, S. (2004). A single target is sufficient to account for the biological effects of the A-factor receptor protein of Streptomyces griseus. J Bacteriol 186, 2206-2211.

Kato, J. Y., Chi, W.-J., Ohnishi, Y., Hong, S.-K. \& Horinouchi, S. (2005a). Transcriptional control by A-factor of two trypsin genes in Streptomyces griseus. J Bacteriol 187, 286-295.

Kato, J. Y., Ohnishi, Y. \& Horinouchi, S. (2005b). Autorepression of AdpA of the AraC/XylS family, a key transcriptional activator in the A-factor regulatory cascade in Streptomyces griseus. J Mol Biol 350, 1226.

Kato, J. Y., Funa, N., Watanabe, H., Ohnishi, Y. \& Horinouchi, S. (2007). Biosynthesis of $\gamma$-butyrolactone autoregulators that switch on secondary metabolism and morphological development in Streptomyces. Proc Natl Acad Sci U S A 104, 2378-2383.

Kawamoto, S., Watanabe, H., Hesketh, A., Ensign, J. C. \& Ochi, K. (1997). Expression analysis of the $s s g A$ gene product, associated with sporulation and cell division in Streptomyces griseus. Microbiology 143, 1077-1086.

Kendrick, K. E. \& Ensign, J. C. (1983). Sporulation of Streptomyces griseus in submerged culture. J Bacteriol 155, 357-366.

Komatsu, M., Takano, H., Hiratsuka, T., Ishigaki, Y., Shimada, K., Beppu, T. \& Ueda, K. (2006). Proteins encoded by the conservon of Streptomyces coelicolor A3(2) comprise a membrane-associated heterocomplex that resembles eukaryotic $G$ protein-coupled regulatory system. Mol Microbiol 62, 1534-1546.

McCue, L. A., Kwak, J., Wang, J. \& Kendrick, K. E. (1996). Analysis of a gene that suppresses the morphological defect of bald mutants of Streptomyces griseus. J Bacteriol 178, 2867-2875.

Ohnishi, Y., Kameyama, S., Onaka, H. \& Horinouchi, S. (1999). The A-factor regulatory cascade leading to streptomycin biosynthesis in Streptomyces griseus: identification of a target gene of the A-factor receptor. Mol Microbiol 34, 102-111.

Ohnishi, Y., Yamazaki, H., Kato, J., Tomono, A. \& Horinouchi, S. (2005). AdpA, a central transcriptional regulator in the A-factor regulatory cascade that leads to morphological development and 
secondary metabolism in Streptomyces griseus. Biosci Biotechnol Biochem 69, 431-439.

Ohnishi, Y., Ishikawa, J., Hara, H., Suzuki, H., Ikenoya, M., Ikeda, H., Yamashita, A., Hattori, M. \& Horinouchi, S. (2008). Genome sequence of the streptomycin-producing microorganism Streptomyces griseus IFO 13350. J Bacteriol 190, 4050-4060.

Onaka, H. \& Horinouchi, S. (1997). DNA-binding activity of the Afactor receptor protein and its recognition DNA sequences. Mol Microbiol 24, 991-1000.

Takano, E. (2006). $\gamma$-Butyrolactones: Streptomyces signalling molecules regulating antibiotic production and differentiation. Curr Opin Microbiol 9, 287-294.

Tomono, A., Tsai, Y., Yamazaki, H., Ohnishi, Y. \& Horinouchi, S. (2005a). Transcriptional control by A-factor of $s t r R$, the pathwayspecific transcriptional activator for streptomycin biosynthesis in Streptomyces griseus. J Bacteriol 187, 5595-5604.

Tomono, A., Tsai, Y., Ohnishi, Y. \& Horinouchi, S. (2005b). Three chymotrypsin genes are members of the AdpA regulon in the A-factor regulatory cascade in Streptomyces griseus. J Bacteriol 187, 6341-6353.

Ueda, K., Oinuma, K., Ikeda, G., Hosono, K., Ohnishi, Y., Horinouchi, S. \& Beppu, T. (2002). AmfS, an extracellular peptidic morphogen in Streptomyces griseus. J Bacteriol 184, 1488-1492.

Ueda, K., Takano, H., Nishimoto, M., Inaba, H. \& Beppu, T. (2005). Dual transcriptional control of amfTSBA, which regulates the onset of cellular differentiation in Streptomyces griseus. J Bacteriol 187, 135142.

Vujaklija, D., Ueda, K., Hong, S.-K., Beppu, T. \& Horinouchi, S. (1991). Identification of an A-factor-dependent promoter in the streptomycin biosynthetic gene cluster of Streptomyces griseus. Mol Gen Genet 229, 119-128.

Yamazaki, H., Ohnishi, Y. \& Horinouchi, S. (2000). An A-factordependent extracytoplasmic function sigma factor $\left(\sigma^{\mathrm{AdsA}}\right)$ that is essential for morphological development in Streptomyces griseus. J Bacteriol 182, 4596-4605.

Yamazaki, H., Ohnishi, Y. \& Horinouchi, S. (2003a). Transcriptional switch on of ssgA by A-factor, which is essential for spore septum formation in Streptomyces griseus. J Bacteriol 185, 1273-1283.

Yamazaki, H., Takano, Y., Ohnishi, Y. \& Horinouchi, S. (2003b). $a m f R$, an essential gene for aerial mycelium formation, is a member of the AdpA regulon in the A-factor regulatory cascade in Streptomyces griseus. Mol Microbiol 50, 1173-1187.

Yamazaki, H., Tomono, A., Ohnishi, Y. \& Horinouchi, S. (2004). DNA-binding specificity of AdpA, a transcriptional activator in the Afactor regulatory cascade in Streptomyces griseus. Mol Microbiol 53, $555-572$.

Edited by: J.-H. Roe 